\title{
Next-generation plate-tectonic reconstructions using GPlates
}

\author{
JAMES A. BOYDEN, R. DIETMAR MÜLLER, MICHAEL GURNIS, \\ TROND H. TORSVIK, JAMES A. CLARK, MARK TURNER, \\ HAMISH IVEY-LAW, ROBIN J. WATSON, AND JOHN S. CANNON
}

\subsection{Introduction}

Plate tectonics is the kinematic theory that describes the large-scale motions and events of the outermost shell of the solid Earth in terms of the relative motions and interactions of large, rigid, intcrlocking fragments of lithosphere called tectonic plates. Plates form and disappear incrementally over time as a result of tectonic processes. There are currently about a dozen major plates on the surface of the Earth, and many minor ones. The present-day configuration of tectonic plates is illustrated in Figure 7.1. As the interlocking plates move relative to each other, they interact at plate boundaries, where adjacent plates collide, diverge, or slide past each other. The interactions of plates result in a variety of observable surface phenomena, including the occurrence of earthquakes and the formation of large-scale surface features such as mountains, sedimentary basins, volcanoes, island arcs, and deep ocean trenches. In turn, the appearance of these phenomena and surface features indicates the location of plate boundaries. For a detailed reviciv of the theory of plate tectonics, consult Wessel and Müller (2007).

A plate-tectonic reconstruction is the calculation of positions and orientations of tectonic plates at an instant in the history of the Earth. The visualization of reconstructions is a valuable tool for understanding the evolution of the systems and processes of the Earth's surface and near subsurface. Geological and geophysical features may be "embedded" in the simulated plates, to be reconstructed along with the plates, enabling a researcher to trace the motions of these features through time. Even a single static reconstruction can reveal an illuminating configuration of surface or subsurface features. A time-sequence of reconstructions may be used to animate the motions of plates, producing kinematic and other time-derivative information, which in turn can provide insight into the geodynamic processes of the near subsurface and deeper Earth.

Plate-tectonic reconstructions are useful in a variety of contexts, such as research in geology, geophysics, and palcobiology; exploration for mineral and hydrocarbon 


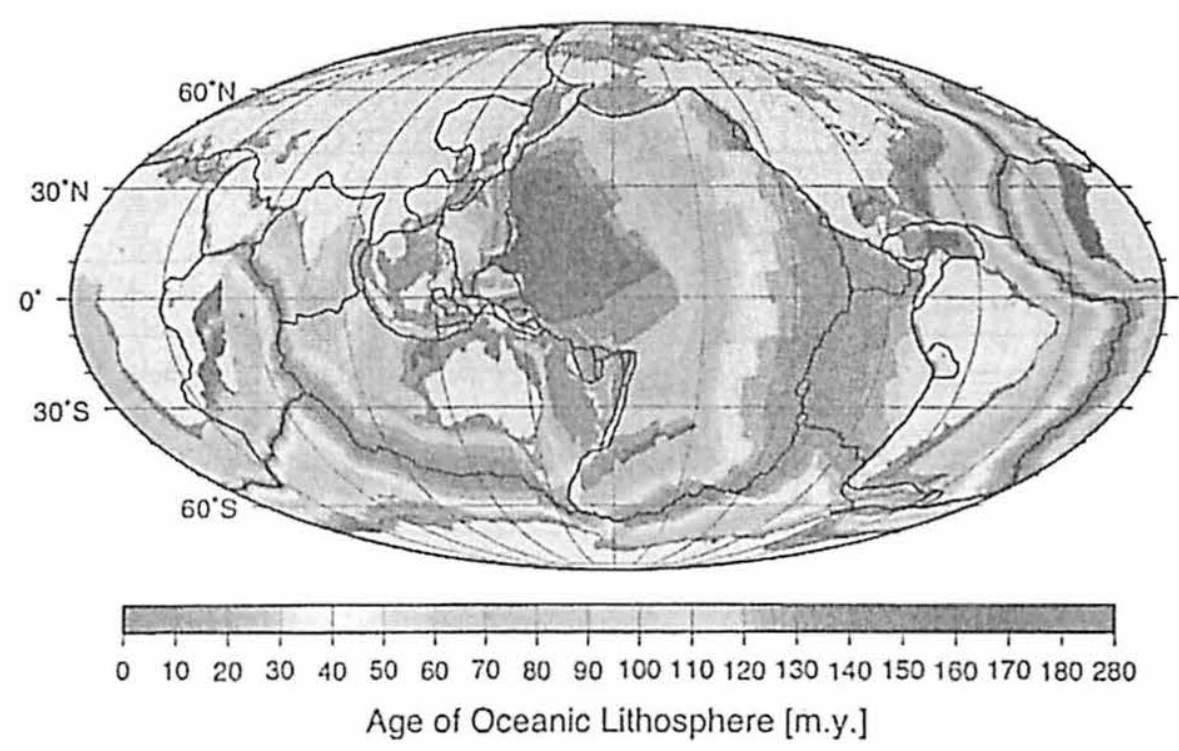

Figure 7.1. The age of the oceanic lithosphere, with plate boundaries shown in black and continents in light gray. See color plates section.

resources; modeling climate change and sea-level change over geological timeperiods, including reconstructing paleotopography, paleovegetation patterns, and paleosurface temperatures to constrain these models (Herold et al., 2008; Herold et al., 2009); and providing kinematic boundary conditions for geodynamic models (e.g., Liu et al., 2008). The ability to reconstruct continents is crucial in any software that is intended to study and explore the distant geological past.

The capability of modern, open-source software has lagged behind the requirements for plate-tectonic reconstructions, which we have attempted to overcome with GPlates, ${ }^{1}$ a desktop software application for the calculation and interactive visualization of plate-tectonic reconstructions. Building upon this foundation of reconstructions, GPlates offers a suite of integrated, interactive functionality for the study of the geological history of the Earth: the visualization of plate-tectonic processes and the dynamics of the upper mantle; the incorporation, reconstruction, and editing of geological, geophysical, and paleogeographic data within a plate-tectonic context; and the real-time graphical manipulation of the plate-motion models which dictate reconstructions. The main window of the GPlates user-interface is illustrated in Figure 7.2 and Figure 7.3.

GPlates can display a reconstruction for a single instant in geological time (in Figure 7.2 and Figure 7.3) or animate a sequence of reconstructions over a userspecified geological time-period. Time-derivative information such as plate-motion velocity fields and flow-lines may be calculated on-the-fly. A user may view reconstructions on either a "flattened-out" geographic map in a variety of 2-D

1 GPlates web site: www.gplates.org. 


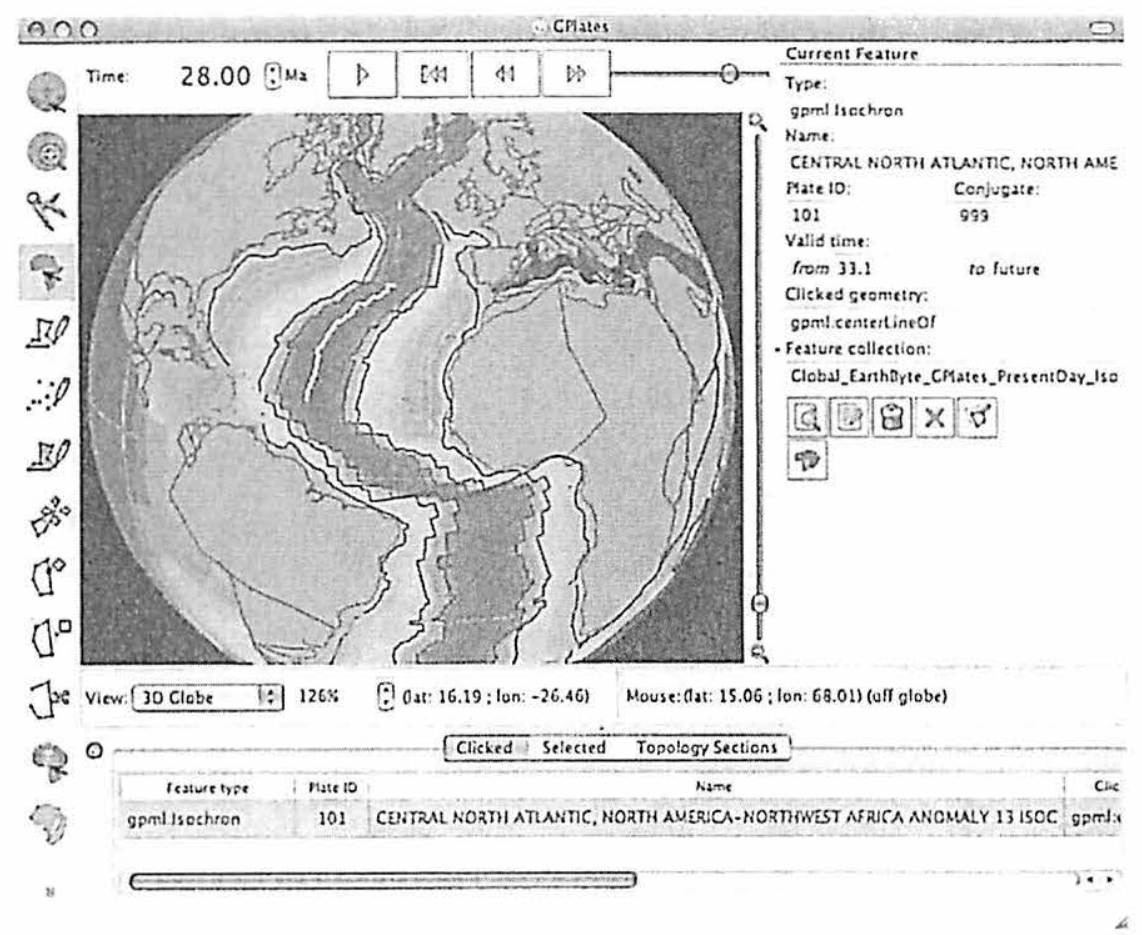

Figure 7.2. The GPlates main window on MacOS X, displaying a reconstruction at $28 \mathrm{Ma}$ (million years ago). The user has clicked on an Isochron feature. See color plates section.

map projections, or an orthographic projection of a 3-D globe (as if observing the Earth from space). Reconstructions may be viewed and manipulated interactively, or they may be exported from GPlates as instantaneous data snapshots (for importation into other geospatial software programs) or as 2-D vector-graphics images in the Scalable Vector Graphics (SVG) format.

The goals motivating the development of GPlates include the need to visualize the reconstruction of geological, geophysical, and paleogeographic data in a variety of formats, including raster data; ${ }^{2}$ to link plate kinematics to geodynamic models; to serve as an interactive client in a grid-computing network; to facilitate the production of high-quality paleogeographic maps; to serve as a freely shared desktop application and software platform, useful both in its own right (as a ready-to-use, stand-alone software product) and as the foundation for new features and specialized functionality to be built on top of the GPlates computational and visualization infrastructure.

GPlates is developed by an international team of scientists and software developers at the EarthByte Project ${ }^{3}$ in the School of Geosciences at the University of

2 A raster is a two-dimensional rectangular grid of values distributed over a spatial extent. Rasters will be discussed in greater detail below.

${ }^{3}$ EarthByte web site: www.earthbyte.org. 


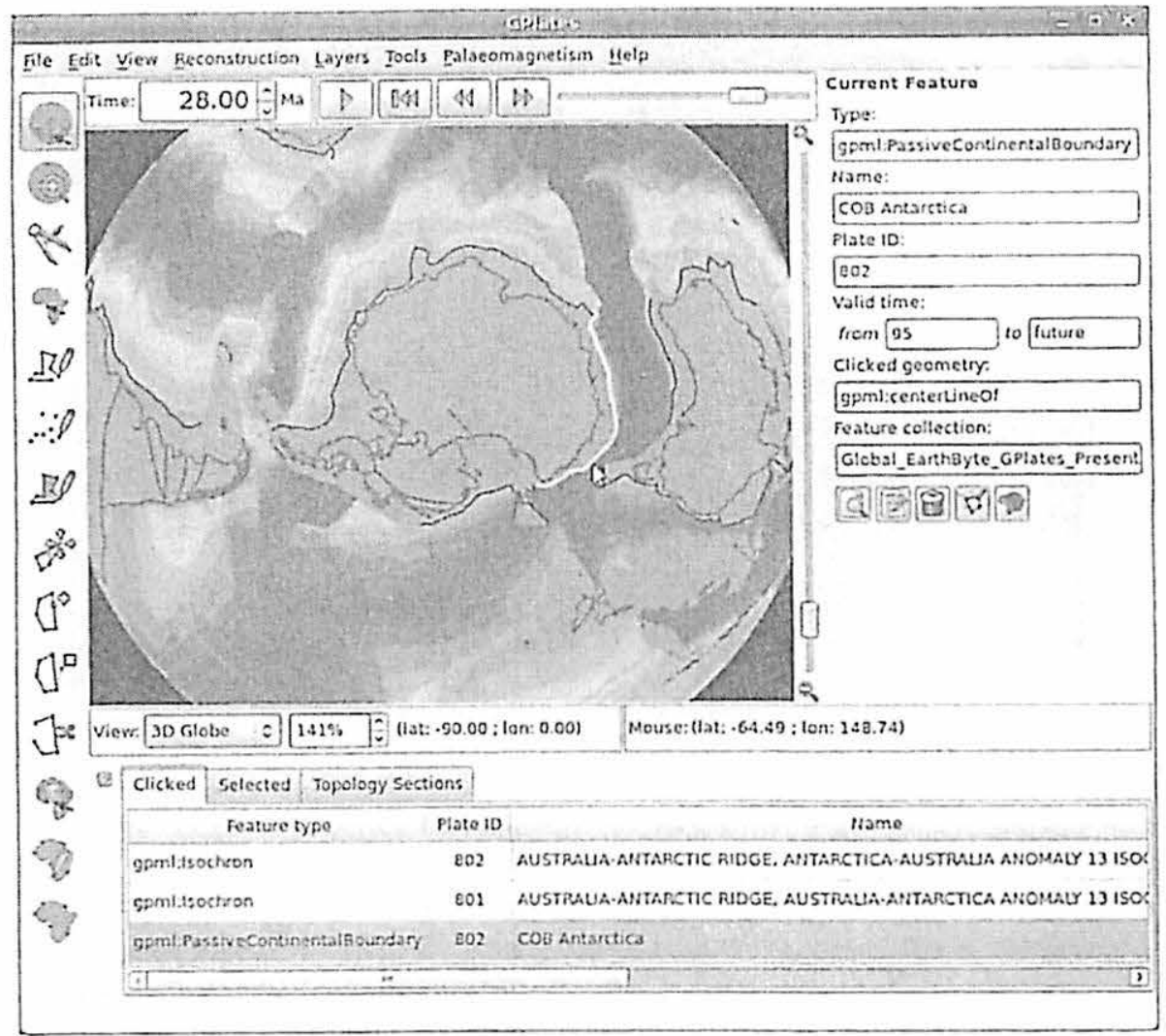

Figure 7.3. The GPlates main window on Linux, displaying a reconstruction at $28 \mathrm{Ma}$. The view of the globe may be re-oriented interactively by dragging on the globe with the mouse. See color plates section.

Sydney, the Division of Geological and Planetary Sciences ${ }^{4}$ (GPS) at Caltech, and the Center for Geodynamics at the Norwegian Geological Survey ${ }^{5}$ (NGU). Freely sharable, royalty-free data for use with GPlates are made available by collaborating scientists at these three institutions.

This chapter introduces GPlates in two parts: Section 7.2 is a survey of the advances in functionality and data-representation that GPlates brings to the domain of interactive plate-tectonic reconstruction software; Section 7.3 is a conceptual overview of the plate-motion model that GPlates uses to calculate reconstructions. Together, these two sections offer an introduction to the foundations and innovations of GPlates.

\subsection{Advancing the state of plate-tectonics software}

Purpose-built interactive visualization software for plate-tectonic reconstructions has existed since the 1980s. The functionality of such software has generally

4 GPS web site: www.gps.caltech.edu. ${ }^{5}$ NGU web site: www.ngu.no/no/. 
included a number of key features: the ability to display a reconstruction for a user-specified geological time; the ability to calculate new reconstructions interactively, by "dragging" reconstructed plates to new locations using the mouse; the ability to control the visual presentation of the reconstructed data (generally a choice of color scheme and map projection, as well as fine-grained control over the visibility of different types of data); and the ability to interact with the data, to query or edit attributes, by "clicking" the visible data with the mouse.

GPlates offers all the familiar features of existing interactive plate-tectonics software, while incorporating a number of advances such as smooth, multiple-frame-persecond animation of reconstructions; interactive graphical manipulation of reconstructions, with continuous real-time feedback and automatic in-place modification of the loaded plate-motion model; seamless display of raster data on the surface of the globe; and automated post-processing of reconstructions, enabling new data to be computed on the fly (such as time-dependent, shape-changing plate-boundary polygons assembled from the geometries of other reconstructed data; and plate-motion velocity fields calculated within these plate-boundaries). These enhancements are presented within a modem, intuitive graphical user interface, which runs on Microsoft Windows, Apple MacOS X, and Linux.

\subsubsection{Fast reconstructions and responsiveness}

GPlates offers unprecedented responsiveness as a result of its computationally efficient calculation of reconstructions and its effective use of hardware-accelerated computer graphics. Calculating a reconstruction is a computationally intensive operation. Due to the choice of programming language $(\mathrm{C}++)$ and judicious selection of the internal representations of geometries and geometric reconstruction operators (3-D unit-vectors (Schettino, 1999) and unit-quaternions (Altmann, 1986), respectively), GPlates is able to reconstruct even large datasets in sub-second times, enabling smooth, real-time animations of reconstructions.

Similarly, the use of the cross-platform, hardware-accelerated 3-D graphics library Open $\mathrm{GL}^{6}$ enables efficient graphics performance. The orthographic globe projection is particularly fast, since the internal representation of geometries (as 3-D unit-vectors) is also an efficient input format for OpenGL in this projection. When viewing the Earth as an orthographic globe, the OpenGL graphics updates are sufficiently fast that when the user "drags" the mouse to reorient the rendered globe, the globe is updated without a perceptible delay. This method of interaction manipulating a displayed object like a real-world object, with rapid, incremental

\footnotetext{
6 OpenGL web site: www.opengl.org.
} 
feedback - is called direct manipulation (Shneiderman, 1983), and is one of the factors which makes a user interface seem more "natural" or "intuitive."

\subsubsection{User-firiendly graphical editing capability}

GPlates extends the familiar graphical editing capability of GIS (Geographic Information System) software to the realm of plate-tectonic reconstructions. Operations such as tweaking geometry shapes and manually fine-tuning reconstructed plate positions are interactive graphical operations in the direct manipulation style. As the user manipulates an object displayed on-screen, GPlates provides continuous, immediate feedback about the result of the operation - for example, as the user drags a vertex of a geometry; a "ghost" of the resulting geometry is drawn and updated in "real-time." When the user releases the mouse button to end the drag operation, the ghost geometry replaces the previous version of the geometry, and the modifications are incorporated automatically into the loaded data. GPlates extends the direct manipulation metaphor to enable reconstructed plate positions to be modified in the same manner: A user can drag a plate into a new position to specify the new reconstruction of that plate at that geological time. GPlates will calculate new plate-motion parameters and incorporate them into the plate-motion model automatically.

New geometries may be digitized in GPlates just as they are in familiar interactive drawing software. However, duc to plate-motion, a geometry on a plate will appear at different positions on the Earth at different geological times - so the position at which a geometry should be digitized is a function of geological time. Fortunately, a user of GPlates need not worry about this issue: GPlates allows a user to digitize a geometry at any geological time; the user should simply digitize the geometry at the appropriate position for the current reconstruction time. When the digitization is complete, GPlates will prompt the user for the plate on which the geometry should be located; the coordinates of the geometry will then be adjusted automatically, to compensate correctly for the reconstruction of that plate at that time.

\subsubsection{Internal geometry representation}

In addition to enabling fast reconstruction and graphics performance, the internal representation of geometries in GPlates (as 3-D unit-vectors) avoids the computational problem of "indeterminate" values at the geographic north or south poles: In a 2-D (latitude, longitude) space, the North Pole has a well-defined latitude of $90^{\circ}$, but no determinate longitude. The alternative is to nominate a particular longitude (for example, $0^{\circ}$ ) to be the longitude of the North Pole by convention; however, this results in a discontinuity between the North Pole and all other regions of high 
latitude that are not on the zero-longitude meridian. At best, either of these situations must be handled by special-case code, which slows the ubiquitous geometric calculations and makes the code more complex (and thus, more difficult to maintain); at worst, these situations are handled incorrectly or not at all during geospatial calculations, leaving the program unable to process data that pass over the poles.

When 3-D unit-vectors are used to encode positions on the surface of the Earth, with the dot product used as the measure of proximity, then the North Pole has a well-defined unit-vector of $(0,0,1)$, without any discontinuities around it. This representation similarly liberates GPlates from any representational or computational discontinuities when geometries cross the International Date Line (and the longitude crosses from $+180^{\circ}$ to $-180^{\circ}$, or vice versa) - or indeed, any other meridian. As a result, GPlates is able to represent data that pass over the poles or cross the Date Line (as illustrated in Figure 7.3), without requiring any special preprocessing of the data (such as cutting geometrics into multiple pieces, or inserting extra vertices at $-180^{\circ}$ and $+180^{\circ}$ ), making GPlates a truly "global" plate-tectonics application.

\subsubsection{Effective representation of continuous fields}

Interactive plate-tectonics software has traditionally modeled geological entities as strictly vector geometries (points, lines, curves or polygons) with associated attributes. Vector geometries are well suited to the representation of location-based measurements (using points), boundaries and contour lines (using lines or curves), and the outlines of enclosed regions (using polygons). However, a continuous field of values distributed over an area - such as the interpolated age and calculated spreading-rate of the ocean floor (Müller et al., 2008), or the dynamic topography of the mantle (Steinberger, 2007) - is not well represented by a spatially discrete vector geometry. Such a field is better represented in raster form.

A raster is a two-dimensional rectangular grid of values distributed over a spatial extent. Each element of the raster is a rectangular cell that contains some scalar or vector values. The cells are contiguous (i.e., there are no gaps between them) and non-overlapping, which makes rasters well suited to the representation of continuous fields in an unambiguous fashion. Each cell contains a sampling of the field at the location of that cell. Since the grid structure of a raster is implied, a raster is a more space-efficient representation than the vector-geometric multi-point equivalent. There are also well-known compression methods for rasters, further increasing the efficiency.

GPlates is able to display rasters on the surface of the globe - both rasters of "raw" data (in which the value in each cell is a physical value, such as the measured seafloor depth or the calculated seafloor spreading-rate) and pre-rendered color 
images (in which the value of each cell is the color of a pixel). The first type of raster is generally the unprocessed result of some geospatial computation or measurement; the second type of raster is either a photographic image, or a computational result or measurement that has already been processed for visualization by some other software. A raster may cover either the entire surface of the globe or a user-specified rectangular surface extent.

GPlates is able to load color images stored in the widely used JPEG image format, as well as raw data rasters stored in the widely used NetCDF format. In the near future, GPlates will be able to display georeferenced rasters in the GeoTIFF and georeferenced JPEG formats.

GPlates is able to load rasters either as single, static images that do not change as the reconstruction time is varied or as frames in a timc-indexed raster sequence. The latter are loaded collectively and treated as a single "time-dependent raster" which changes as a function of geological time. The former method of loading is used for a "normal" raster that is not part of a time-secuence and is not intended to change over time, while the latter is used for a pre-prepared raster sequence, which GPlates will animate.

\subsubsection{Precise, consistent definition of data attributes}

Representing heterogeneous real-world geospatial information can be a complex issue. At one extreme, if we attempt to express all information uniformly in terms of a fixed set of attributes, we risk both missing important attributes that are relevant to only some types of information, as well as coercing our users' view of the world to fit into those attributes. At the other extreme, if wc accept any combination of attributes and attribute types, there is no guidance about which attributes are recommended, expected, or required by the software. As a result, attributes will be chosen ad hoc from project to project, hindering attempts to share data between projects. Both of these extremes have been employed in widely used plate-tectonics software.

GPlates avoids these pitfalls through the definition of its own precise abstract information model for data to be handled by GPlates. Lee (1999) defines an information model as "a representation of concepts, relationships, constraints, rules, and operations to specify data semantics for a chosen domain of discourse." Lee continues: "The advantage of using an information model is that it can provide sharable, stable, and organised structure of information requirements for the domain context."

An information model is used to obtain "a single, integrated definition of the data within an enterprise that is unbiased toward any single application of data and independent of how the data are physically stored or accessed," which "provides a consistent definition of the meanings and interrelationship of the data in order to share, integrate, and manage the data." (Lee, 1999) 
The GPlates Geological Information Model (GPGIM) is the formal specification of all data in GPlates. It specifies the geologic, geophysical, and paleogeographic entities that GPlates simulates, the conceptual building blocks that GPlates defines, and the processing and simulation constraints to which GPlates adheres. It serves as the primary definition of the data that must be representable in GPlates, in order for GPlates to be able to present and process plate-tectonic reconstructions as the users of GPlates require. The current GPGIM specification is available ${ }^{7}$ on the web site of the EarthByte e-research project. ${ }^{\mathrm{s}}$

Each geological and tectonic entity is described precisely in formal modeling terms, without concern for how the entity will be represented within software or encoded in data files. This liberates the description of data from concerns of fileformat representation; the model of each entity has exactly the attributes it needs, no more and no less. The precise definition avoids any ambiguity about which attributes are expected or required.

The GPlates software developers have worked closely with collaborating geologists to create, refine and evolve the GPGIM specification over time. The GPGIM is currently able to represent both the legacy geological data of the collaborating scientists, as well as various next-generation data for state-of-the-art functionality being developed in GPlates (such as time-dependent geometries defined by geospatial topological networks). The GPGIM continues to be extended to incorporate new types of data - recent additions include the paleomagnetic data-types from the PMAG portal of the Magnetics Information Consortium (MaglC) database ${ }^{9}$ on the EarthRef web site. ${ }^{10}$

\subsubsection{Ability to represent next-generation data}

Traditional plate-tectonics data have consisted of vector geometries and "simple type" attributes such as strings, integers, and floating-point numbers. On their own, these building blocks are insufficient to represent the next-generation data calculated and processed by GPlates, such as attribute values which vary as functions of geological time; geological timescales; structured edit-history metadata; evolving topological plate boundaries; and dynamic flowlines.

The last two examples are particularly illustrative of the paradigm shift that is being pioneered by GPlates: Rather than containing static, pre-calculated or prepopulated geometries and geospatial values, these two data types instead encode templates for geospatial calculations. To be specific, they contain parameters for calculations that are performed in GPlates as automated post-processing of a

7 GPGIM specification on the EarthByte tveb site: www.earthbyte.org/Resources/GPGIM/public/.

8 EarthByte e-rescarch projecl: www.carthbyte.org. ${ }^{9}$ MagIC databasc: hitp://earthref.org/MAGIC/.

10 EarthRef: http://earthref.org/. 
reconstruction, to generate results that are based upon the other data loaded in GPlates, including the plate-motion model. For example, a topological plate boundary lacks any static geometry of its own; at each reconstruction time, GPlates calculates a topological geometry by linking together the reconstructed geometries of other data. This topological geometry is a time-dependent, shape-changing polygon that encloses a region corresponding to a tectonic plate (Gurnis et al., 2010). As the tectonic plate changes shape over time, so too does the polygon.

A topological plate boundary is "data," because a user defines the topology by selecting geometries from other reconstructed data, to specify geometry sections from which a plate-boundary polygon should be assembled. This topology configuration is stored in a data file like any other data, and the assembly of the polygon occurs only when the topological boundary data are loaded, after the component sections have been reconstructed. After the polygon has been assembled, it is rendered on the globe just like any other geometry, enabling the topological plate boundary to be visualized and manipulated just like any other geospatial data.

Given a mesh of points, GPlates can use these plate-boundary polygons to "cookie cut" the mesh points and allocate them to plates. At each reconstruction time, GPlates can then calculate a velocity for each mesh point, by first calculating the angular velocity of the relevant plate motion; the result will be a plate-motion velocity field for that reconstruction time. In addition to being visualized in GPlates, such a platemotion velocity field may be exported, to be used as a kinematic boundary condition to a geodynamic mantle-convection model such as CitcomS (Spasojevic et al., 2009).

The key to being able to represent these next-generation data in GPlates is the ability to describe and represent structured information in attributes - in particular, the ability to nest structured information inside other structured information to an arbitrary degree. Such nesting of structured information enables GPlates to represent arbitrarily complex information by composing structures like functions are composed in mathematics. The GPGIM provides a precise specification of this structured information, and GPlates implements this specification to represent these next-generation data internally - but this does not solve the problem of how to store these data on disk between GPlates sessions, or how to transport and exchange these data between systems. For this purpose, the developers of GPlates have specified a new file format for plate-tectonics data called GPML, the GPlates Markup Language. " GPML is XML-based, which enables it to define and contain arbitrarily nested structure. In fact, the structure of GPML mirrors the structure of the GPGIM exactly: A GPML document is an XML serialization ${ }^{12}$ of GPGIM data.

"GPML: www.gplates.org/gpml.html.

12 Serialization is the process of converting an in-memory object into a sequence of bytes so it can be stored in a file or transmitted across a network. 


\subsubsection{Superior interoperability of data}

The GPGIM and GPML are both based upon GML, the Geography Markup Language (Cox et al., 2004; Lake et al., 2004). GML is a modeling language for geographic systems, as well as an encoding specification for the serialization of GML data to XML documents. GML is intended to provide a standardized, interoperable base for defining application-specific geospatial languages. The GML specification is defined and maintained by the Open Geospatial Consortium. GML was adopted as an ISO standard in 2007.

GML defines information model building blocks including geometries; temporal primitives (time-instants and time-periods); units of measure; and coordinate reference systems (Lake, 2005). These building blocks ease the process of interoperability both by providing recognizable, well-defined data components and by standardizing the XML representations of these components, to simplify the parsing of foreign data. GML also provides an approach to modeling, the "feature-oriented" approach, which adds additional consistency to the structure of data (Zhang et al., 2003). The GPGIM adopts the feature-oriented modeling approach and builds upon the GML primitives, to maximize interoperability with other GML-based languages such as GeoSciML (Sen and Duffy, 2005).

The Web Feature Service (WFS) specification (Vretanos, 2005) defines a web service interface to query, transmit, and manipulate geospatial data across the Internet or other networks. GML is the default encoding for the transmission of geospatial data to and from WFS servers; as an application schema of GML, the GPGIM can be used to define geospatial features in WFS communications, enabling GPlates data to be queried and transmitted across WFS networks. Future GPlates development efforts will implement WFS client operations in GPlates itself, to enable GPlates to query and transmit geospatial data as an interactive client in a WFS-compatible grid-computing network.

Finally, by building upon GML, the GPGIM will also benefit from future additions to GML. For example, Cox and Richard (2005) describe a GML-compatible model of geological timescales, which will be incorporated into GPlates in the future.

\subsubsection{Availability of the softwave source code}

Perhaps one of the most significant advantages of GPlates is that the GPlates source code can be freely shared as open-source software. As a result, anyone can enhance GPlates with new technology as it becomes available; anyone can extend GPlates with new functionality; anyone can fix any bugs in GPlates which might be discovered; and anyone can port GPlates to new computer systems if desired. 
GPlates is distributed under the GNU General Public License (GPL), version $2,^{13}$ which grants to every person who receives a copy of GPlates the permission to reproduce, adapt or distribute GPlates as long as the source code of any resulting copies or adaptations are also made available under the same "share alike" licensing scheme.

\subsection{Reconstructions and plate motions in GPlates}

\subsubsection{Modeling tectonic plates}

In contrast to traditional GIS software, GPlates was designed and built from the ground up to be geotemporally aware and to handle plate-tectonic reconstructions. Thus, a core concept in GPlates is that geospatial entities move around through geological time due to plate tectonics or other deep-Earth geological processes. This motion is expressed in the plate-motion model, which is used to reconstruct geospatial entities to their past locations. The plate-motion model is itself composed of data that may be loaded, saved, visualized, and edited in GPlates.

The majority of geological, geophysical, and palcogeographic entities modeled by GPlates are embedded in tectonic plates, so will move around with these plates. However, a key aspect of the GPlates plate-motion model is that there is no fundamental "tectonic plate" entity with a well-defined boundary. This is because the boundaries of tectonic plates are not always well defined; their relative motions more clearly differentiate adjacent plates. Furthermore, the shapes and extents of plates change over geological time, as plate motion causes plates to diverge and converge, with new oceanic lithosphere being produced from the mantle or old oceanic lithosphere being consumed by the mantle. Plates can stretch, compress, and otherwise deform. Finally, the set of tectonic plates has not remained constant over time: Plates can break up and fuse due to tectonic processes.

Thus, the GPlates plate-motion model instcad focuses on relative motion. The key concept is the plate ID: an integer identifier that associates all entities that move together as if they were embedded in a single rigid plate. The plate-motion model describes the relative motions of entities with different plate IDs; cvery reconstructable entity is given a plate ID to describe its motion. Many of the plate IDs do correspond to rigid subregions of plates, but other plate IDs correspond to non-plate entities that move relative to plates (such as mid-ocean ridges and hotspots). Plate IDs are not "hard coded" or otherwise pre-determined: The allocation of plate IDs is up to the user.

13 GPL version 2: www.gnu.org/licenses/old-licenses/gpl-2.0.html. 


\subsubsection{Finite rotations and Euler poles}

The hypothesis of plate tectonics states that tectonic plates are assumed to be internally rigid (Wessel and Müller, 2007). Euler's Displacement Theorem (also called Euler's Rotation Theorem) states that any displacement of a rigid body, such that one point of the rigid body remains fixed in space, is a rotation about some axis through that fixed point (Beatty, 1986). If the Earth is approximated as a sphere, then any motion of a rigid tectonic plate on the surface of the Earth may be described geometrically as a displacement of the plate about the fixed center of the Earth, and thus, as a rotation of the plate about an axis through the center of the Earth. Cox and Hart (1986) refer to such rotations as finite rotations. Finite rotations are commonly used in plate-tectonics literature to describe plate motions on a large scale.

By analogy with the Earth's geographic north pole (the point at which the Earth's own rotation axis intersects with the surface of the planet), a common means of specifying the axis of a finite rotation is to specify the point at which the axis intersects with the surface of the Earth. This intersection point is called the Euler pole of the finite rotation. The Euler pole and its spherical antipode are the only two points on the surface of the Earth that are not moved to a new location by the finite rotation.

The position of the Euler pole is described conveniently as a (latitude, longitude) pair, meaning that a finite rotation can be specified by a scalar triple: the latitude of the Euler pole, the longitude of the Euler pole, and the angle of rotation. Finite rotations are commonly published in this form in plate-tectonics literature; this is also the form used to enter finite rotations into GPlates, bcfore they are converted automatically to the unit-quaternion representation used internally.

\subsubsection{Relative motions and the rotation hierarchy}

GPlates uses a plate-motion model representation in which plate motions are described in terms of relative rolations between pairs of plates. For example, in the GPlates-compatible 2008 EarthByte Global Rotation Model (Müller et al., 2008), the South American plate (also known by the abbreviation "SAM," with plate ID 201) moves relative to the African plate ("AFR," 701), as does the Antarctic plate ("ANT," 802), while the Australian plate ("AUS," 801) moves relative to the Antarctic plate. This is illustrated in Figure 7.4.

Thus, the full plate-motion model is a directed graph of rotations between pairs of plates. Every plate in the model moves relative to some other plate or is the "fixed reference frame" relative to which some other plates move. In general, large plates are described relative to other large plates, and smaller plates are described relative to their nearest large plates. This tree-like structure is called the rotation hierarchy. 


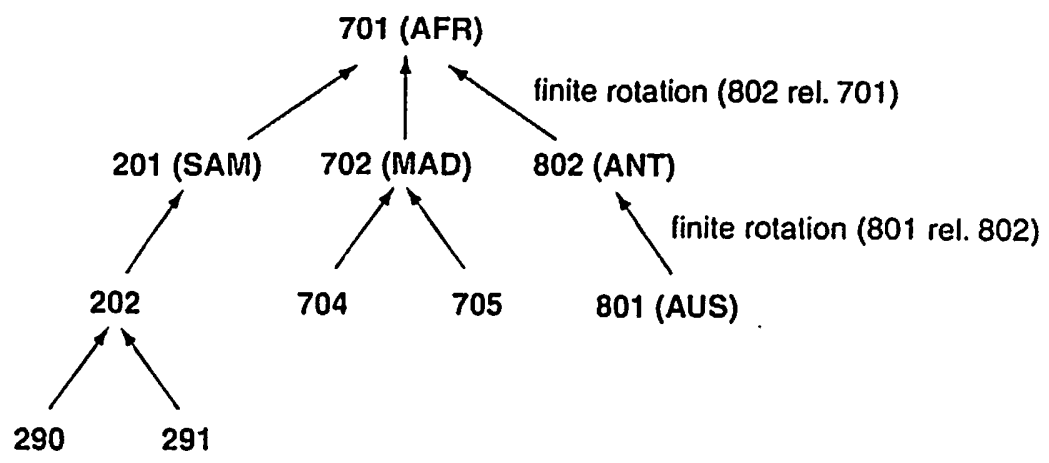

Figure 7.4. A small subset of the hierarchy of relative rotations used in GPlates.

Even if there is actually no relative motion between some pair of plates for some period of time, the plates may nevertheless be related by the identity rotation (a rotation of zero angle, which effects no change of position or orientation). The identity rotation does not determine a unique axis of rotation, and so is not strictly speaking a finite rotation, but since the identity rotation does not change the shape of the plate or move the plate relative to the center of the Earth, there is no geometric problem with allowing identity rotations in the rotation hierarchy.

\subsubsection{Total reconstruction poles}

The relative displacement of adjacent plates may be determined quantitatively by a number of techniques, including the fitting of magnetic anomaly and fracture zone data on the ocean floor by matching conjugate isochrons (e.g., Hellinger, 1981) and the fitting of apparent polar wander curves for continents (e.g., Van der Voo, 1993). These changes may be described quantitatively using finite rotations, though Kearey and Vine emphasize (1990): "although the relative motion between two plates can be described in terms of an angular rotation about a pole, the plates do not necessarily follow the route predicted by a single rotation, and may have followed a much more complex path."

Thus, given a measurement of the relative displaccment that occurred between two plates over some time-period, simply scaling the angle of the finite rotation (in order to interpolate the displacement over time) will not necessarily yield an accurate history of the relative positions of the plates over time. Measurements of intermediate displacements are necessary to build an accurate picture - the more measurements, the better.

The finite rotation that displaces a plate from its present-day position to its reconstructed position at some instant in the past is called a total reconstruction pole (Cox and Hart, 1986) (henceforth TRP). A TRP is associated with a fixed plate, a moving plate, and a particular instant in geological time: Such a TRP reconstructs 
the moving plate to its position relative to the fixed plate at that time. The TRP for the present day is the identity rotation. A sequence of TRPs for a particular moving plate is used to describe the plate's motion through time by sampling its relative displacement at key instants in the past; TRPs for intermediate times may be interpolated according to the mathematical rules described by Cox and Hart (1986).

\subsubsection{Equivalent rotations and absolute reference firmes}

Cox and Hart (1986) outline a simple algebra for composing TRPs of the same geological time. The kcy is to match up fixed plates and moving plates to form a continuous plate circuit through the graph of the rotation hicrarchy. For example, the fixed plate and moving plate of a relative motion may be swapped if the direction of the finite rotation is reversed: The reverse of $B$-relative-to- $A$ yields $A$-relative-to$B$. Similarly, TRPs for the same geological time may be composed if the fixed plate of one TRP is matched to the moving plate of the other: $C$-relative-to- $B$ may be composed with $B$-relative-to- $A$ to give $C$-relative-to- $A$. Using this algebra, a circuit of relative motion may be traced between any two plates in the rotation hierarchy.

GPlates enables the user to specify an "anchored plate" which will be held fixed in the reconstruction. By composing and reversing the appropriate TRPs in the rotation hierarchy, GPlates can trace a plate circuit to any choice of anchored platc from every plate in the hierarchy, to calculate an equivalent rotation for each plate relative to the anchored plate (Ross and Scotese, 1988). These equivalent rotations may be used to reconstruct all the other plates relative to the anchored plate at that geological time. In an animation, the anchored plate would remain still, and all other plates would move relative to it. While this is not necessarily geologically realistic, it is nevertheless useful as a means to study relative motions, and is sufficient for many regional models.

Other uses of plate tectonics - such as using plate motions to provide kinematic boundary conditions to geodynamic mantle-convection models (O'Neill el cl., 2005) require information about the motions of plates relative to the interior of the Earth itself. For this purpose, it is necessary to identify a measurable geological frame of reference that is consistent over tens or hundreds of millions of years - this is termed an absolute reference frame. Examples of absolute reference frames include hotspot reference frames (relative to stationary or slow-moving mantle plumes that rise up through the mantle) and paleomagnetic reference frames (Torsvik and Smethurst, 1999) (relative to the average location of the Earth's magnetic north polc over millions of years).

The plate-motion predictions of different absolute reference frames do not always coincide. Part of this disagreement may be understood in terms of information limitations inherent to each particular type of absolute relerence frame. For example, hotspot reference frames are disadvantaged by a limited number of 


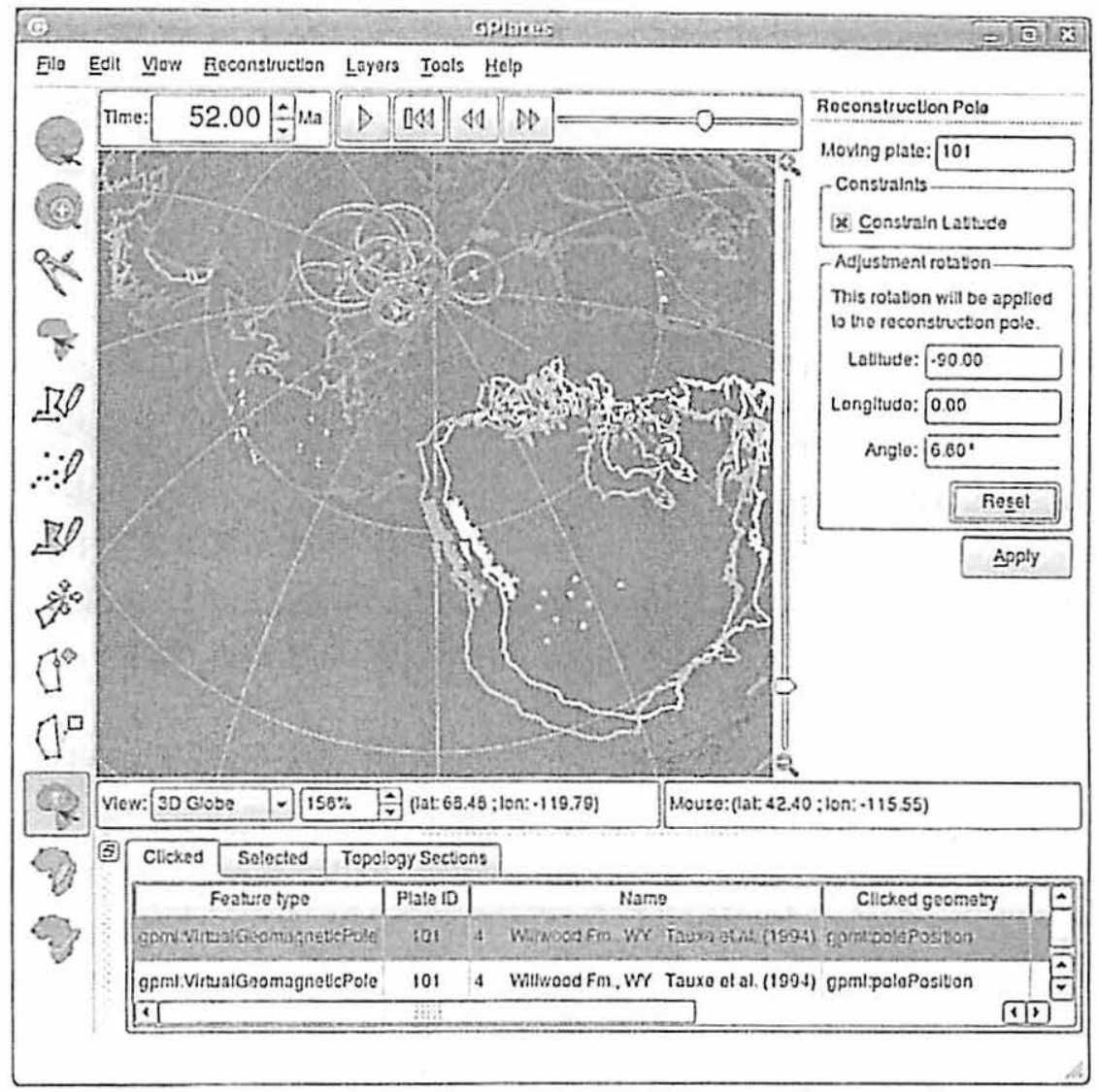

Figure 7.5. Modifying a plate reconstruction in GPlates using the interactive Modify Reconstruction Pole tool. Paleomagnetic data are being used to align the plates, so it is helpful to constrain the latitude of the plate; only the longitude of the plate will change. See color plates section.

hotspots; a lack of hotspots on many plates; and a lack of hotspot data older than 200 million years (Greiner, 1999). In contrast, the main disadvantage of paleomagnetic reference frames is that, due to the axial symmetry of the Earth's magnetic poles, paleomagnetism can only determine the latitude and orientation of a plate, not the longitude (Torsvik and Smethurst, 1999). As a result, a researcher must be able to test a reconstruction hypothesis using different absolute reference frames. Figure 7.5 illustrates how a GPlates user may experiment with an alternate plate reconstruction by varying only the longitude.

In the 2008 EarthByte Global Rotation Model, the relative motions between plates and absolute reference frames are also described using total reconstruction poles; the absolute reference frames have three-digit plate IDs which begin with a " 0 ." A GPlates user may specify an absolute reference frame as the "anchored plate," to observe the motions of the plates relative to this geological frame of reference. This is illustrated in Figure 7.6. 


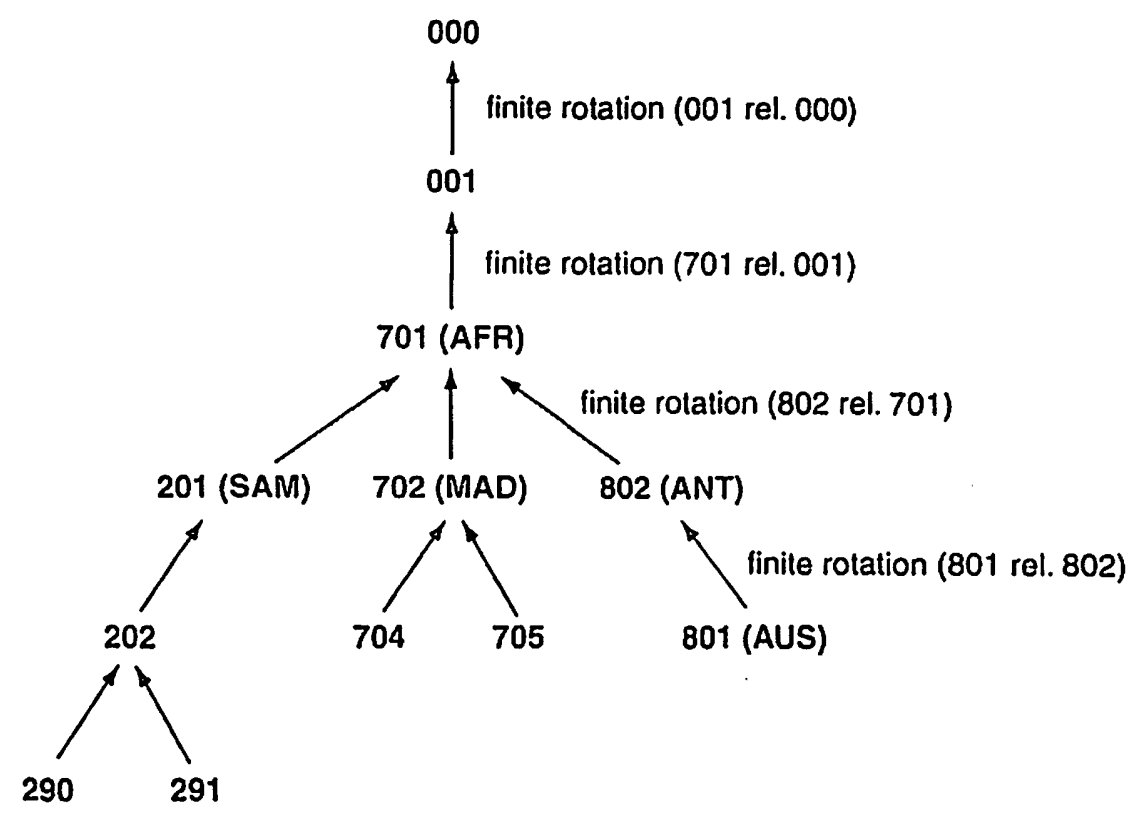

Figure 7.6. Relative rotations with an absolute reference frame, as used in GPlates.

\subsection{Conclusions}

Offering all the familiar functionality of traditional plate-tectonics reconstruction software, and based upon a solid theoretical foundation of finite rotations, relative motions and the rotation hicrarchy, GPlates measures up as an interactive plate-tectonics application. In fact, more than simply meeting expectations, GPlates advances the state of the art along several dimensions of functionality. These advances are due to a combination of successful leveraging of technological advancements (OpenGL, Qt, XML, GML) and judicious selection of suitable techniques (such as the internal geometry representation based upon 3-D unit-vectors and unit-quaternions, and the careful modeling of the geological domain).

There are four significant differences in GPlates that set it apart from previous plate-tectonic reconstruction software: The rigorous modeling methodology used to model geological, geophysical and paleogeographic features in the GPGIM and enable GPlates to serve as a component in a larger GML-based infrastructure; the sophisticated information model building blocks which enable expression of advanced and abstract concepts; the paradigm shift of calculation templates for automated post-processing of reconstructions, to enable new data to be computed on the fly based upon plate motions; and finally, the fact that GPlates is released as open-source software, enabling anyone to build upon or modify this foundation, to extend GPlates to meet their needs, and then release their work back to the geology community, further enriching the community in a virtuous cycle. 
Future GPlates development will include the ability to process paleomagnetic features to derive new total reconstruction poles automatically; the ability to read and write GeoSciML documents, and reconstruct GeoSciML features alongside GPGIM features; the ability to ready and write GML-based data, participating in a computing grid as a WFS client; and the ability to reconstruct rasters on plates. These innovations will enable users to investigate alternative fits of the continents interactively, simultaneously displaying and fitting data such as faults, sutures, orogenic belts, terranes, paleomagnetic poles, and raster data such as magnetic or gravity grids, to test hypotheses of supercontinent formation and breakup through time. Users will also be able to derive and test models for the evolution of tectonically complex areas such as the Arctic, the Caribbean and Southeast Asia. The diverse functionality of GPlates, paired with its free availability, will also allow teachers to explore creative ways of integrating plate tectonics into educational activities.

With a flexible, expressive, interoperable information model and the ability to represent calculation templates as data, GPlates leads the arrival of the next generation of interactive plate-tectonics software - a generation in which a plate-tectonics application does not operate in isolation, but as an integrated visualization and processing component within a computational grid; and a platc-tectonic reconstruction is no longer an isolated result, but a single stage in an adaptable workflow.

\section{References}

Altmann, S. (1986). Rotations, Quaternions and Double Groups. Oxford: Oxford University Press.

Beatty, M. F. (1986). Principles of Engineering Mechanics. New York: Springer, 595pp.

Cox, A. and Hart, R. B. (1986). Plate Tectonics: How /l Works. Ox ford: Blackwell Scientific Publications.

Cox, S. J.D. and Richard, S. M. (2005). A formal model for the geologic time scale and global stratotype section and point, compatible with geospatial transfer standards. Geosphere, 1: 119-137.

Cox, S.J.D., Daisey, P., Lake, R., Portele, C., and Whiteside, A., eds. (2004). OpenGIS Implementation Specification \#03-105rl: OpenGIS Geography Markup Language (GML) Implementation Specification, version 3.1.1. February 2004.

Greiner, B. (1999). Euler rotations in plate-tectonic reconstructions. Computers \& Geosciences, 25(3): 209-216.

Gurnis, M., Turner, M., Zahirovic, S. et al. (2010). Plate reconstructions with continuously closing plates. Computers and Geosciences, in review.

Hellinger, S.J. (1981). The uncertainties of finite rotations in plate tectonics. Journal of Geophysical Research, 86: 9312-9318.

Herold, N., Seton, M., Müller, R. D., You, Y., and Huber, M. (2008). Middle Miocene tectonic boundary conditions for use in climate modcls. Geochemistry Geophysics Geosystems, 9, Q10009, doi:10.1029/2008GC002046. 
Herold, N., You, Y., Müller, R. D., and Sdrolias, M. (2009). Climate model sensitivity to changes in Miocene paleo-topography. Australian Journal of Earth Sciences, 56(8): 1049-1059.

Kearey, P. and Vine, F.J. (1990). Global Tectonics. Oxford: Blackwell Scientific Publications.

Lake, R., Burggraf, D. S., Tminić, M., and Rae, L. (2004). Geography Markup Language: Foundation for the Geo-Web. Chichester, UK: John Wilcy \& Sons.

Lakc, R. (2005). The application of geography markup language (GML) to the geological sciences. Computers \& Geosciences, 31(9): 1081-1094.

Lee, Y.T. (1999). Information Modeling: From Design to Implementation. National Institute of Standards and Tec/mology. Available at www.mel.nist.gov/msidlibrary/ doc/tina99im.pdf, last accessed 2009-04-08.

Liu, L. J., Spasojevic, S., and Gurnis, M. (2008), Reconstructing Farallon plate subduction beneath North America back to the Late Cretaceous. Science, 322: 934-938.

Müller, R. D., Sdrolias, M., Gaina, C., and Roest, W. R. (2008). Age, spreading rates and spreading asymmetry of the world's occan crust. Geochemistiy Geophysics Geosystems, 9(4): 1-19, doi:10.1029/2007GC001743.

O'Neill, C. J., Müller, R. D., and Steinberger, B. (2005). On the uncertainties in hotspot reconstructions, and the significance of moving hotspot reference frames. Geochemistry Geophysics: Geosystems, 6: doi:10.1029/2004GC000784.

Ross, M. I. and Scotese, C. R. (1988). A hierarchical tectonic model for the Gulf of Mexico and Caribbean region. Tectonophysics, 155: 139-168.

Schettino, A. (1999). Polygon intersections in spherical topology: Application to plate tectonics. Computers \& Geosciences, 25(1): 61-69.

Sen, M. and Duffy, T. (2005). GeoSciML: Development of a gencric GeoScience Markup Language. Computers \& Geosciences, 3(9): 1095-1103.

Shneiderman, B. (1983). Direct manipulation: A step beyond programming languages. IEEE Computer; 16(8): 57-69.

Spasojevic, S., Liu, L., and Gurnis, M. (2009). Adjoint models of mantle convection with seismic, plate motion and stratigraphic constraints: North America since the Late Cretaceous. Geochemistry, Geophysics Geosystems, 10, Q05W02, doi:10.1029/ $2008 \mathrm{GC} 002345$.

Steinberger, B. (2007). Effects of latent heat release at phase boundaries on flow in the Earth's mantle: Phase boundary topography and dynamic lopography at the Earth's surface. Physics of the Earth and Planetary Interiors, 164: 2-20.

Torsvik, T.H. and Smethurst, M. A. (1999). Plate tectonic modelling: virtual reality with GMAP. Computers \& Geosciences, 25(4): 395-402.

Van der Voo, R. (1993). Paleomagnetism of the Atlantic, Tethys and Iapetus Oceans. Cambridge: Cambridge University Press.

Vretanos, A. P., ed. (2005). OpenGIS Implementation Specification \#04-094rl: Web Feature Service Implementation Specification, version 1.1.0. May 2005.

Wessel, P. and Müller, R.D. (2007). Plate tectonics. In Treatise on Geophysics. Vol. 6. Amsterdam: Elsevier, pp. 49-98.

Zhang, C., Peng, Z. R., Li, W., and Day, M. J. (2003). GML-based interoperable geographical databases. Available at wwwv.ucgis.org/summer03/studentpapers/chuanrongzhang.pdf. 

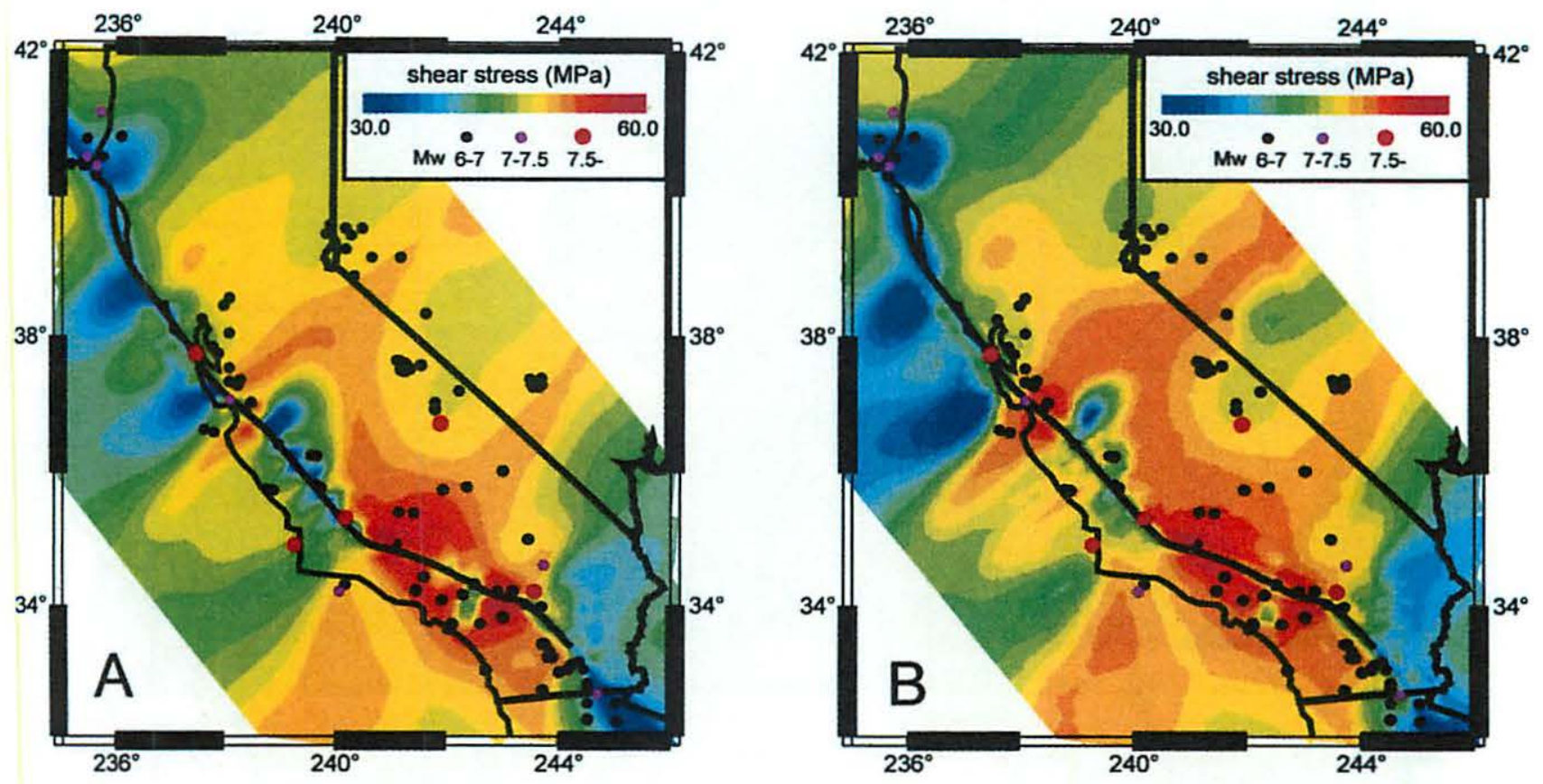

Plate 14. Predicted background shear stress. (A) Cumulative shear stress after 40000 model years of repeated ruptures along various segments of the SAF. (B) Steady-state background shear stress predicted by the long-term faulting model where fault slip is simulated by continues plastic creep ( $\mathrm{Li}$ and Liu, 2006). Dots are earthquakes. (See Figure 6.13.)

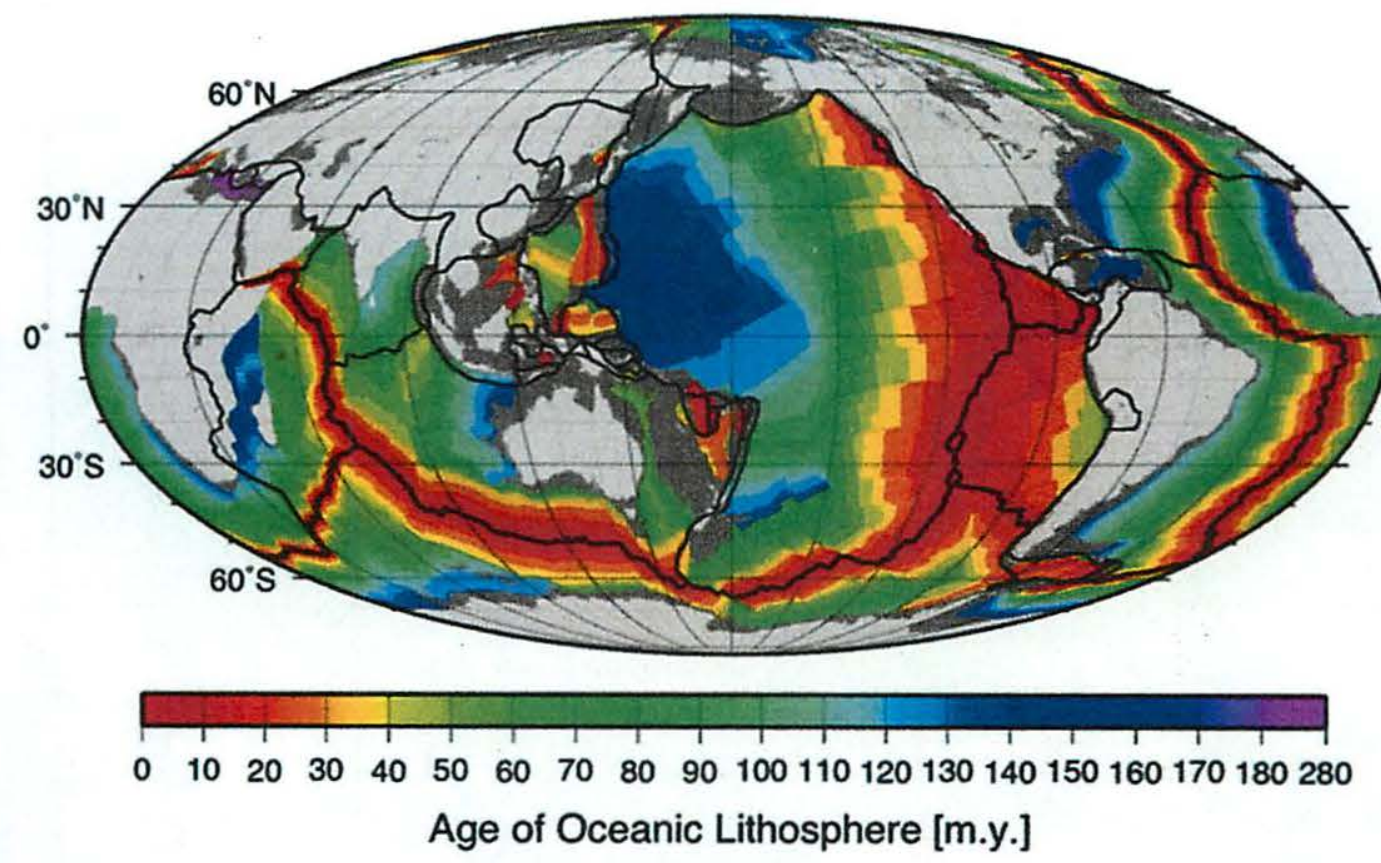

Plate 15. The age of the oceanic lithosphere, with plate boundaries shown in black and continents in light gray. (See Figure 7.1.) 


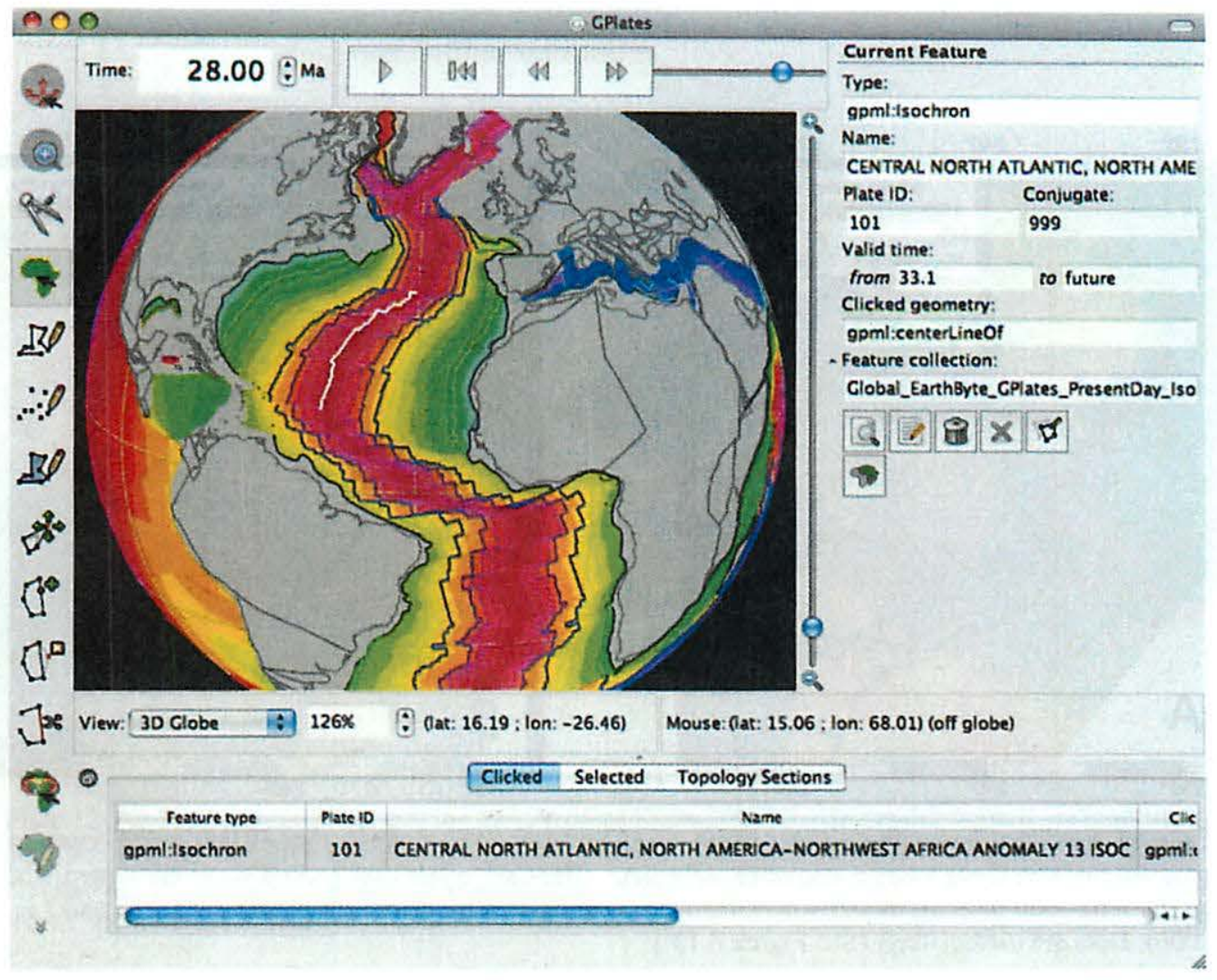

Plate 16. The GPlates main window on MacOS X, displaying a reconstruction at $28 \mathrm{Ma}$ (million years ago). The user has clicked on an Isochron feature. (See Figure 7.2.)

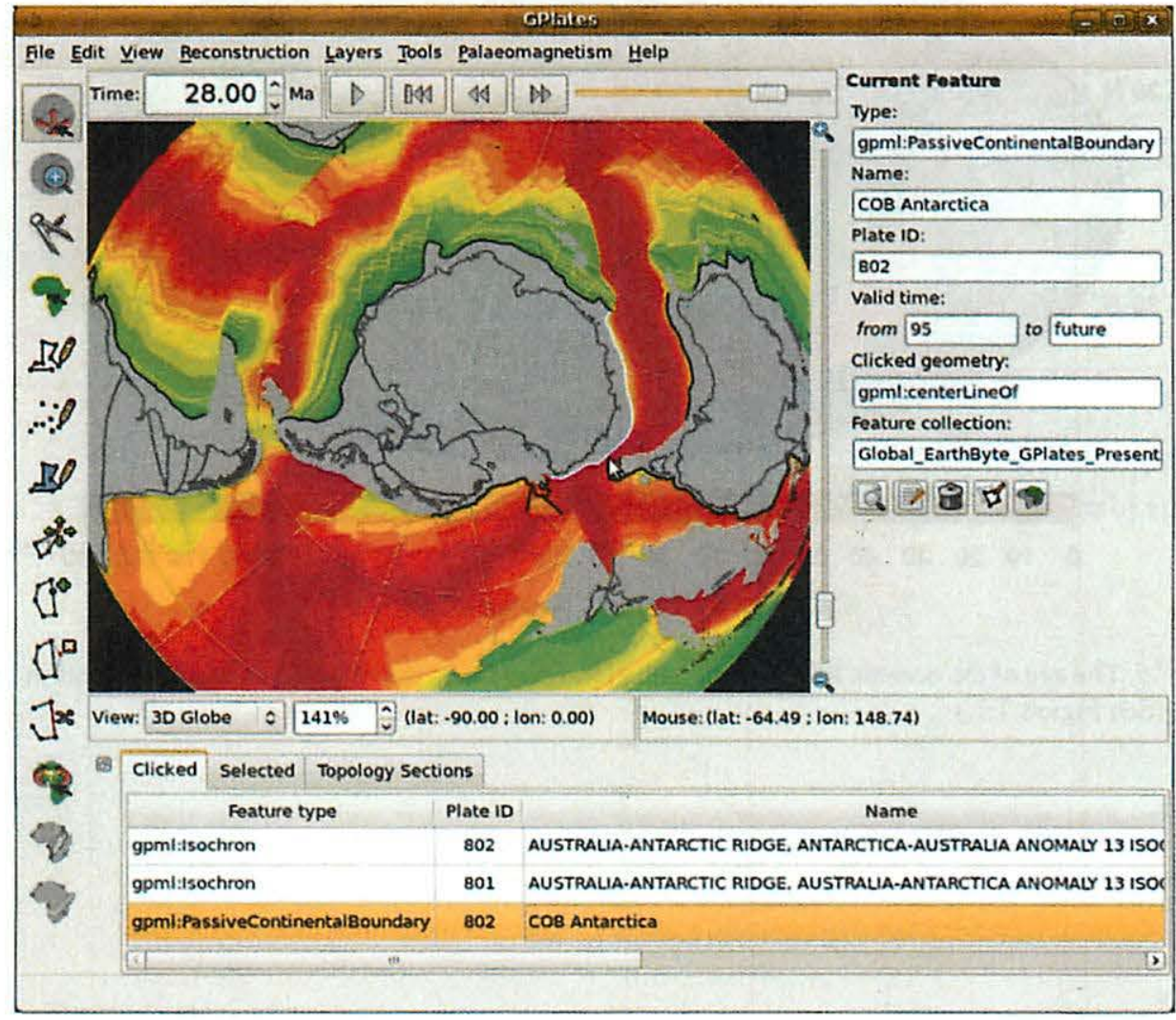

Plate 17. The GPlates main window on Linux, displaying a reconstruction at $28 \mathrm{Ma}$. The view of the globe may be re-oriented interactively by dragging on the globe with the mouse. (See Figure 7.3.) 


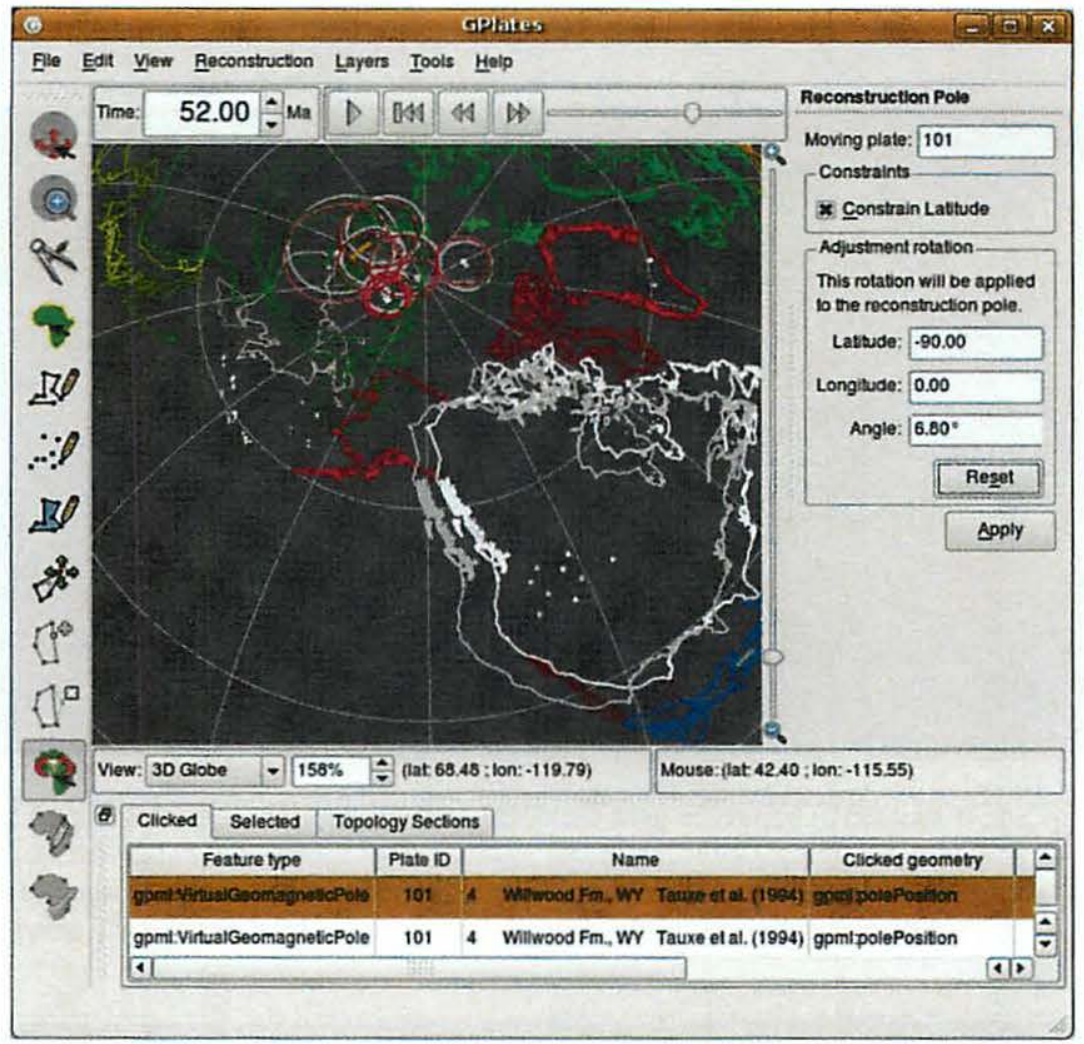

Plate 18. Modifying a plate reconstruction in GPlates using the interactive Modify Reconstruction Pole tool. Paleomagnetic data is being used to align the plates, so it is helpful to constrain the latitude of the plate; only the longitude of the plate will change. (See Figure 7.5.)

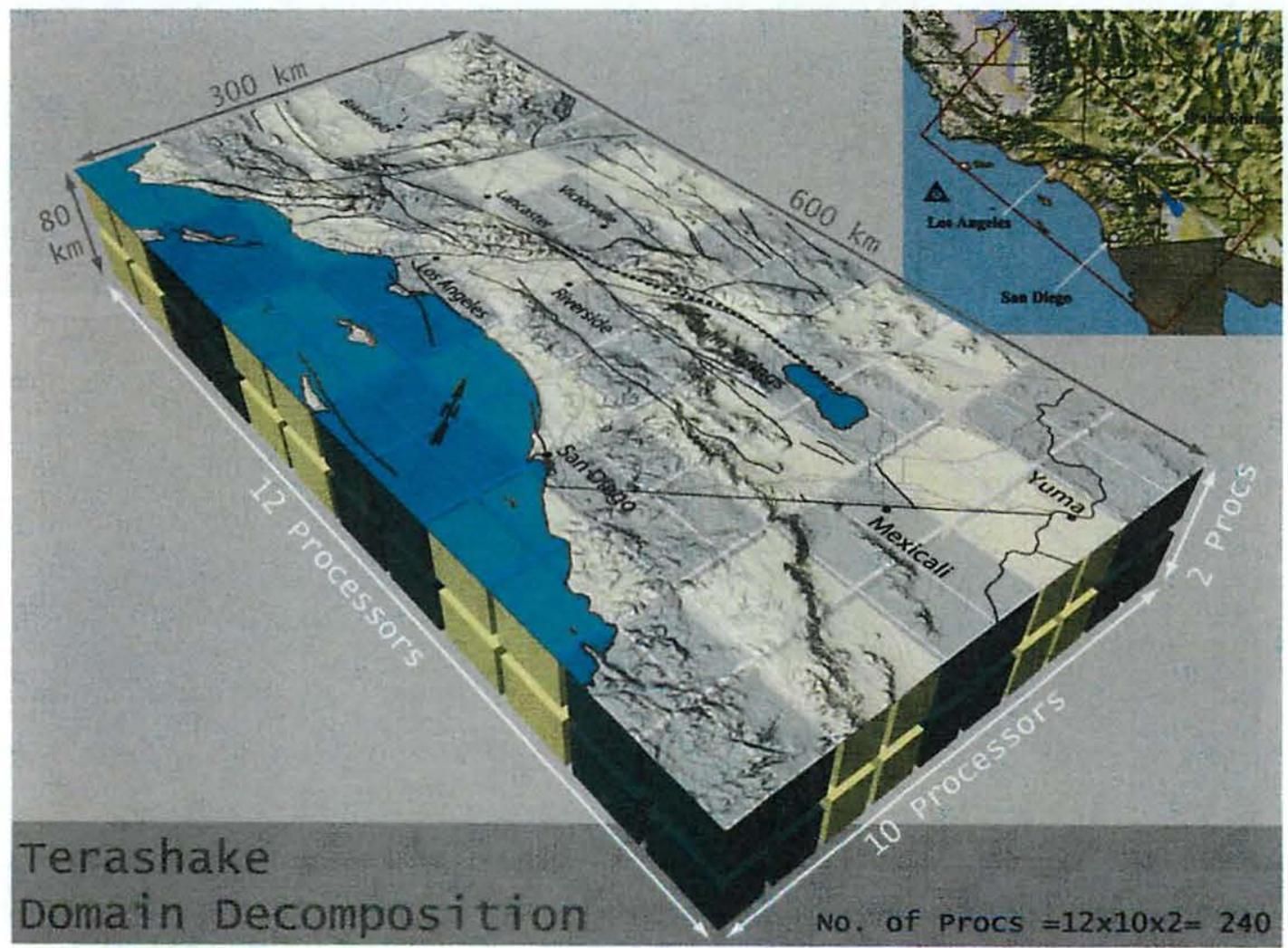

Plate 19. The top right inset shows the geographic location of the simulation region depicted by red rectangle. In the center, the topography, fault lines, and city locations are visible. This also shows the domain decomposition of this region into 240 processors. (See Figure 8.1.) 